\title{
AS CONTRIBUIÇÕES DE CHARCOT E DE MARSDEN PARA O DESENVOLVIMENTO DOS DISTÚRBIOS DO MOVIMENTO NOS SÉCULOS XIX E XX
}

\author{
Hélio A. G. Teive', Jorge A.A. Zavala², Fábio M. Iwamoto², \\ Daniel Sá3, Hipólito Carraro Júnior ${ }^{3}$, Lineu Cesar Werneck ${ }^{4}$
}

\begin{abstract}
RESUMO - Charcot contribuiu significativamente no século XIX na descrição de várias enfermidades neurológicas, em particular na área dos distúrbios do movimento. Charcot contribuiu de forma exponencial na descrição clínica minuciosa da doença de Parkinson, além de introduzir o primeiro tratamento farmacológico. Na área das hipercinesias realizou estudos sobre a síndrome de Tourette, o diagnóstico diferencial dos tremores, das coréias e o estudo inicial sobre startle. Marsden, recentemente falecido, destacou-se no século XX com inúmeras publicações na área dos movimentos anormais.São contribuições seminais os estudos sobre a doença de Parkinson, distonias, mioclonias, tremor essencial, a descrição das síndromes " Painful Legs Moving Toes ", "Gait Ignition Failure" e o "Tremor Primário da Escrita". As contribuições de Charcot no século XIX e de Marsden no século XX na área dos distúrbios do movimento permitem concluir que ambos foram as figuras mais representativas desta área nos últimos dois séculos.
\end{abstract}

PALAVRAS-CHAVE: Charcot, Marsden, distúrbios do movimento.

Contributions of Charcot and Marsden to the development of movement disorders in the $19^{\text {th }}$ and $20^{\text {th }}$ Centuries

\begin{abstract}
Charcot described many neurological diseases in the $19^{\text {th }}$ century, particularly in movement disorders. Charcot contributed in the clinical description of Parkinson's disease, and introduced its first pharmacological treatment. He also studied the hyperkinesias, e.g. of Tourette syndrome, differential diagnosis of tremors, dystonias, choreas and startle disease. Marsden, who died recently, was an exponent in the study of Movement Disorders, with many publications in this field in the $20^{\text {th }}$ century. His most important contributions are definitions and classifications of movement disorders, such as Parkinson's disease, dystonia, myoclonus, essential tremor, the description of the syndromes "Painful Legs Moving Toes", "Gait Ignition Failure" and "Primary Writing Tremor". The contributions of Charcot in the $19^{\text {th }}$ century and Marsden in the $20^{\text {th }}$ century to the movement disorders allow us to conclude that both of them were the most representative icons in this field in the past two centuries.
\end{abstract}

KEY WORDS: Charcot, Marsden, movement disorders.

J-M Charcot é considerado o fundador da primeira escola neurológica do mundo, "pai da neurologia", e o primeiro professor de doenças do sistema nervoso, no século $\mathrm{XIX}^{1,2}$. A sua contribuição na descrição de várias enfermidades neurológicas, como a esclerose lateral amiotrófica, a esclerose múltipla, a doença de Charcot-Marie-Tooth, é sobejamente conhecida $^{1-3}$. Outra faceta também importante do mestre de Salpêtrière foi a sua contribuição no estudo e definição dos distúrbios do movimento 4 .

C.D. Marsden foi um dos mais brilhantes cientistas biomédicos, sendo um dos neurocientistas mais citados no mundo e líder da escola neurológica britânica na segunda metade do século $X X^{5-9}$. Suas contribuições na área dos distúrbios do movimento colocaram-no como o mais importante pesquisador dos distúrbios do movimento deste século ${ }^{10}$.

O objetivo do presente estudo é o de revisar as principais contribuições destes dois mestres, nos séculos $X I X$ e XX, na área dos movimentos anormais.

\section{MÉTODOS}

Foram revisadas várias publicações acerca das contribuições de J-M Charcot no século XIX e de C.D. Marsden

Setor de Distúrbios do Movimento, Serviço de Neurologia, Hospital de Clínicas, Universidade Federal do Paraná (UFPR), Curitiba PR, Brasil: ${ }^{1}$ Professor Assistente; ${ }^{2}$ Residente em Neurologia; ${ }^{3}$ Médico Neurologista; ${ }^{4}$ Professor Titular.

Recebido 5 Fevereiro 2001, recebido na forma final 30 Abril 2001. Aceito 10 Maio 2001. 
no século $X X$, na área dos distúrbios do movimento. A pesquisa foi realizada através do Index Medicus e da Internet, com a Pubmed (National Library of Medicine).

\section{DISCUSSÃO}

Charcot e Marsden, vultos eméritos das escolas francesa e inglesa de neurologia, desenvolveram seus estudos neurológicos em diferentes situações históricas. Pode-se dizer que o primeiro criou a especialidade de neurologia e procurou, através de método de estudo anátomo-clínico, definir as principais enfermidades neurológicas, retirando-as da completa obscuridade (Fig 1). Charcot valorizou sobremaneira a intuição (com empirismo) e a experiência pessoal clínico-patológica ${ }^{1-4}$. Marsden iniciou seus estudos na área neurológica já encontrando-a em sua quase-maturidade e buscou estudar os diferentes distúrbios de movimentos de forma racional, experimental, com uma demonstração científica de causa e efeito (Fig 2). Mesmo atuando por diferentes métodos e em diferentes épocas, ambos os pesquisadores contribuíram significativamente para o desenvolvimento da área dos distúrbios do movimento.

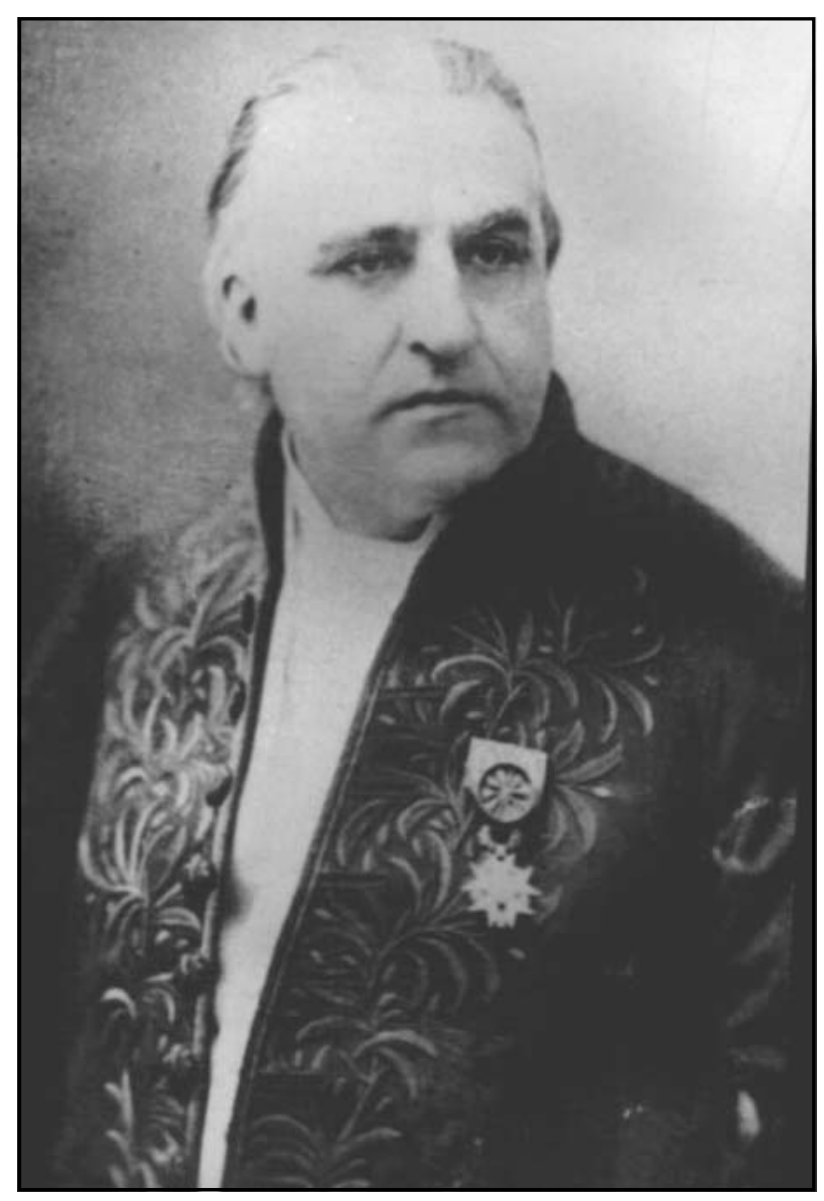

Fig 1. J-M CHARCOT.
Charcot foi o primeiro neurologista a criar um serviço de documentação fotográfica dos pacientes com enfermidades neurológicas, em particular com distúrbios de movimentos, posteriormente publicadas na "iconographie photographique de la Salpêtrière" ${ }^{\prime \prime}$.

A contribuição de Charcot no estudo da doença de Parkinson (DP) foi de grande importância ${ }^{11}$. Foi Charcot quem sugeriu o nome "doença de Parkinson" para a enfermidade "paralisia agitante". Por outro lado Charcot acrescentou várias contribuições pessoais na descrição do quadro clínico, definindo a presença dos chamados sinais cardinais da doença, apresentando critérios para o diagnóstico diferencial e também sugerindo o primeiro tratamento para a enfermidade ${ }^{1-4,11}$.

Charcot definiu a presença do tremor como sintoma cardinal da DP, referindo-se ao início unilateral, geralmente no membro superior, observando-se no período de repouso. Realizou uma série de estudos comparativos diferenciando o tremor parkinsoniano de outros, como o tremor da esclerose múltipla (do tipo cerebelar) e o tremor senil. Outra característica

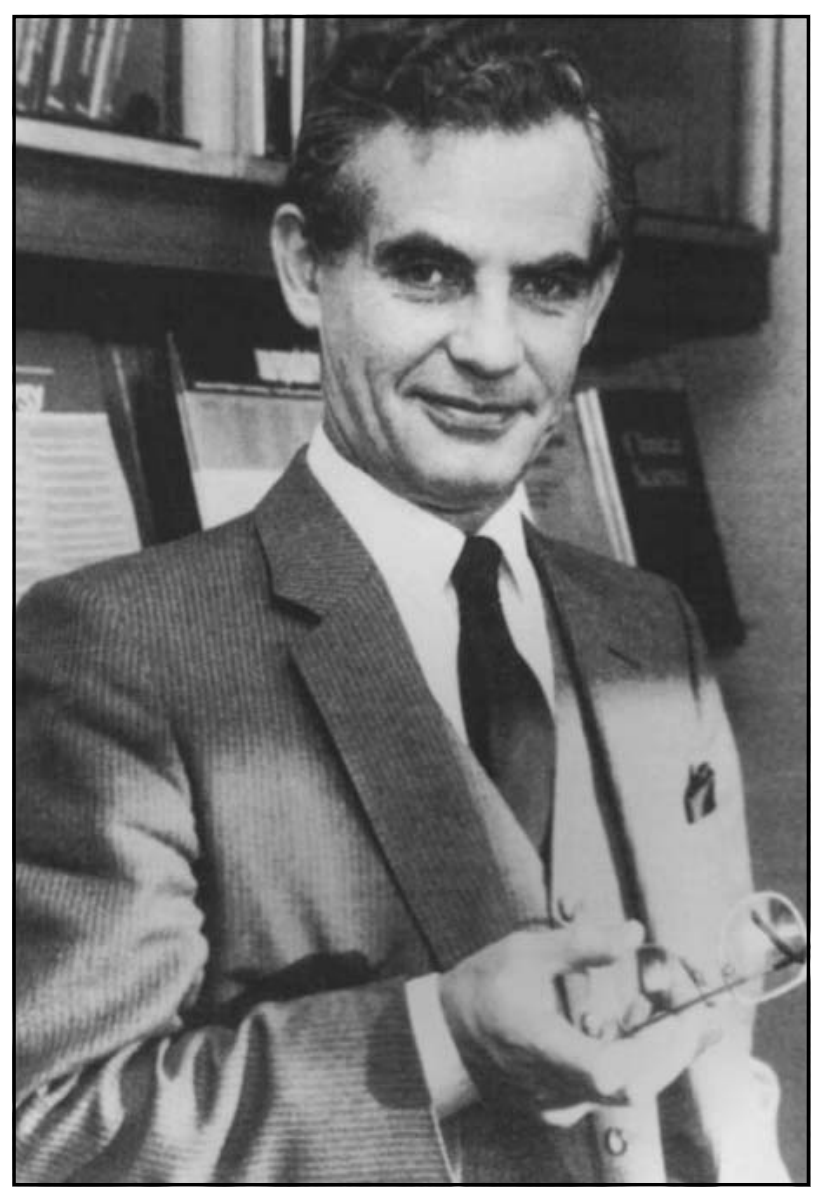

Fig 2. C.D. MARSDEN. 
definida por Charcot quanto ao tremor da DP foi o acometimento da cabeça, considerada por ele raro ${ }^{2-11}$.

Charcot também definiu a presença de alterações posturais na DP, com a descrição da atitude peculiar do corpo e dos membros, com o olhar fixo, e as tendências de propulsão e retropulsão. A escrita peculiar dos pacientes, com micrografia, bem como a disartria e a hipofonia foram características também descritas. Outra característica de extrema importância na DP, definida por Charcot, e que não havia sido descrita por James Parkinson, foi a rigidez muscular. A presença de fraqueza muscular foi contestada por Charcot, e foi descrita a presença de bradicinesia. Charcot também discordou da descrição original de James Parkinson quanto à preservação das funções corticais superiores na doença. Ele descreveu a presença de disfunção cognitiva, fato este hoje bastante conhecido. Charcot foi o primeiro neurologista a sugerir um tratamento para a DP. Em 1877 ele iniciou o tratamento com uso de hioscinamida, um precursor dos alcalóides da beladona, com propriedades anticolinérgicas ${ }^{1-4,11}$.

No grupo das hipercinesias, Charcot contribuiu significativamente para o estudo da síndrome de Tourette (ST), enfermidade por ele assim denominada "la maladie de tics convulsives") ${ }^{1-4}$. Além de ser o mentor intelectual de Gilles de la Tourette, Charcot descreveu vários sintomas da enfermidade e das suas co-morbidades. Em primeiro lugar, ele separou a ST da síndrome dos franceses saltadores do Maine, da Latah e da Myriachit que haviam sido definidas por Tourette como enfermidades conjuntas. Charcot separou a ST também das coréias e da histeria. Charcot classificou os diferentes tipos de tiques, sugeriu a evolução e o prognóstico para a doença e um fator hereditário, além de destacar a presença do transtorno obsessivo-compulsivo ("folie du doute") 1-4.

Charcot discutiu, de forma não aprofundada, as diferenças entre as coréias de Sydenham (CS) e de Huntington (DH), definindo a presença de hipotonia na CS (coréia mole), sem relatar o caráter hereditário da $\mathrm{DH}$ e da presença de disfunção cognitiva ${ }^{1-4}$.

Outra área de interesse discutida por Charcot foi a das ataxias locomotoras, em especial a ataxia de Friedreich. Nesta entidade, Charcot estudou a localização da lesão degenerativa na medula espinhal (coluna posterior) diferenciando-a de outras enfermidades (sífilis, por exemplo) e das chamadas ataxias verdadeiras (cerebelares) ${ }^{1-4}$.

A Tabela 1 apresenta de forma sintética as principais contribuições de Charcot na área dos distúrbios do movimento.

Os estudos de Marsden envolveram áreas de neurofisiologia, neurofarmacologia e neuropsicologia ${ }^{5-9}$. O seu interesse na fisiologia do movimento e no funcionamento dos gânglios da base culminaram no célebre e classico trabalho "The Mysterious Motor Function of the Basal Ganglia"12. Marsden contribuiu de forma muito significativa na definição e classificação de diferentes distúrbios de movimentos $^{5-9}$.

Marsden contribuiu de forma exponencial no estudo da DP, com estudos sobre a bioquímica da doença, particularmente dos defeitos do complexo 1 da mitocôndria e os estudos sobre o estresse oxidativo na sua etiopatogenia. Outra importante contribuição foi o apoio para a criação do banco de cérebro de Londres, que propiciou vários estudos de extrema importância ${ }^{5-9}$.

$\mathrm{Na}$ área das hipercinesias, a contribuição do professor Marsden foi contudo mais significativa. Os estudos sobre as distonias foram extremamente relevantes. Ele "trouxe ordem ao caos que eram as distonias", nas palavras do professor D. Brooks ${ }^{7}$.

Marsden definiu que as distonias não eram fenômenos histéricos e estudou mais profundamente o grupo das distonias focais, como o blefaroespasmo, além do torcicolo e da cãimbra do escrivão. Marsden foi o primeiro pesquisador a sugerir o tratamento

Tabela 1. Principais contribuiçôes de Charcot para o desenvolvimento dos distúrbios do movimento.

- Sugestão do nome "doença de Parkinson", com definição clínica meticulosa da doença e utilização do primeiro tratamento medicamentoso.

- Diagnóstico diferencial dos tremores.

- Definição clínica, sugestão do nome "Síndrome de Tourette" e suas co-morbidades.

- Diagnóstico diferencial das coréias de Sydenham e Huntington.

- Estudo e classificação das ataxias locomotoras. 
Tabela 2. Principais contribuiçôes de Marsden para o desenvolvimento dos distúrbios do movimento.

- Estudos sobre o funcionamento dos gânglios da base.

- Doença de Pakinson: Estudos sobre a bioquímica da doença.

- Definição clínica das distonias e introdução do tratamento com altas doses de anticolinérgicos.

- Estudo neurofisiológico e classificação das mioclonias.

- Descrição da síndrome "Painfull Legs and Moving Toes".

- Descrição da entidade "Tremor Primário da Escrita".

- Descrição da síndrome "Gait Ignition Failure".

- Estudos sobre controle postural, equilíbrio, apraxias e distúrbios da marcha.

das distonias com altas doses de anticolinérgicos para pacientes jovens ${ }^{5}$.

A contribuição de Marsden para o estudo das mioclonias foi também muito significativa, com a classificação e definição das mesmas, a descrição de diferentes formas de mioclonias, como a mioclonia reticular e a cortical reflexas, e a mioclonia pós-hipó$x_{i c a^{5-9}}$. Marsden também estudou com profundidade os tremores, tendo publicado uma classificação de tremor essencial, que tornou-se referência obrigatória neste campo ${ }^{5}$. Outras áreas de interesse do professor Marsden foram as do equilíbrio, postura, distúrbios de marcha e as apraxias.

Marsden também contribui com a descrição de algumas enfermidades, como a síndrome "painful legs and moving toes", "o tremor primário da escrita" e a síndrome "gait ignition failure"5-9.

Muitas outras contribuições científicas do professor Marsden encontram-se publicadas na literatura médica mundial, perfazendo a fabulosa quantidade de mais de 1100 trabalhos científicos, sendo considerado o mais produtivo e mais citado neurocientista de nossa época ${ }^{5-10}$.
A Tabela 2 demonstra de forma resumida as principais contribuições de Marsden na área dos distúrbios do movimento.

Charcot e Marsden, trabalhando em diferentes épocas, com diferentes métodos, contribuíram de forma exuberante para o desenvolvimento da área dos distúrbios de movimentos nos séculos XIX e XX.

\section{REFERÊNCIAS}

1. Guillain G. J-M Charcot 1825-1893: his life-his work. New York: Paul B Hoeber, 1959.

2. Goetz G, Bonduelle M, Gelfand T. Charcot: constructing neurology. New York: Oxford University Press, 1995.

3. Goetz G. Charcot: the clinician, the tuesday lessons. New York; Raven Press, 1987.

4. Teive HAG. Charcot e os distúrbios do movimento. Striatum 1997;1:2-4.

5. Journal Neurol Neurosurg Psychiatry. Special Supplement, June, 1989.

6. Lees AJ. In Memoriam C. David Marsden. Mov Disord 1999;14:3-5.

7. Brooks DJ. Obituary Professor C. David Marsden 1938-1998. J Neurol Neurosurg Psychiatry 1999;66:2.

8. Lang AE. In Memoriam Professor Charles David Marsden. Neurology 1999;52:14-15.

9. Fahn S. Professor C-David Marsden. Arch Neurol 1999;56:119.]

10. Teive HAG. In Memoriam C.D. Marsden. Striatum 1998;4:2-3.

11. Teive HAG. O papel de Charcot na doença de Parkinson. Arq Neuropsiquiatr 1998;56:141-145.

12. Marsden $C D$. The mysterious motor function of the basal ganglia: the Robert Wartemberg Lecture. Neurology 1982;32:514-539. 